\title{
Two Hand Gesture Recognition Using Stereo Camera
}

\author{
Rustam Rakhimov Igorevich, Pusik Park, Jongchan Choi, and Dugki Min
}

\begin{abstract}
This paper proposes method for gesture recognition by two hands. In this research stereo camera was used to calculate depth map of the frame. Based on depth information precise tracking of the hands was applied. Depth map calculates in hardware level. Oversensitivity of camera to light generates horizontal noise. Especially appeared horizontal noise affects on tracking two hands located in same altitude. In this paper we suggest masking method to avoid horizontal noise in software level.
\end{abstract}

Index Terms-Hand tracking, stereo camera, two hand gesture recognition, horizontal noise, dynamic programming, depth map.

\section{INTRODUCTION}

A Tracking and recognition human gesture becomes popular these days. The reason these technologies becomes popular is because it can be applied into many different fields easily and efficiently. Gesture recognition easily can be applied to the web services, smart home systems, robot manipulation and games. That is why tracking non-rigid motions from sequential videos have been a great interest to the computer vision community [1]. Nowadays ubiquitous systems intend to make peoples life better. It requires technologies to interact with computer using some markers or gloves. But interaction without using any kind of additional devices is considered as an advanced and high technological point in Human Computer Interaction (HCI). That is why HCI has been occupying almost all fields [2].

In this research we are using special stereo camera with hardware implemented depth map. As an output from stereo camera we will receive three images: left, right and depth map image [3]. The main reason why we use stereo camera in this research is that it can simulate human binocular vision [4], therefore it gives the ability to capture three-dimensional images, a process known as stereo photography. Stereo camera can also be used for making stereo views and 3D pictures for movies, or for range imaging.

Our work is dedicated to track two hands in frame. For that we are referring to [5], where we are calculating threshold value for the filtering by depth grayscale value. Usually in gesture recognition systems based on stereo camera,

Manuscript received October 14, 2012; revised November 29, 2012.

Rustam Rakhimov Igorevich is with the Korea Electronics Technology Institute, Korea (e-mail: rustam@ keti.re.kr).

Pusik Park, was with the Korea Electronics Technology Institute, Korea $\mathrm{He}$ is now with the SoC Research Platform Center (e-mail: parksik@keti.re.kr).

Jongchan Choi is with the Korea Electronics Technology Institute, Korea. $\mathrm{He}$ is now Vice President in System IC R\&D department. (e-mail: choijc @keti.re.kr).

Dugki Min is Professor in Department of Computer Science, Konkuk University, Korea (e-mail: dkmin@konkuk.ac.kr). threshold of the depth map filtering has a fixed value. It means if subject who wants to be recognized with his hands gestures in some environment, subject has to come close to certain fixed distance to the camera. Environment of using this system can be smart home systems, smart kitchen or just game playroom. We are suggesting another solution where distance between camera and subject becomes flexible. That is why the environments using our algorithm can be considered as flexible gesture recognition systems.

Suggested advantage in this paper makes gesture recognition distance more flexible and also it give change to use additionally one more hand. With two hands the range of commands what subject can perform multiplicatively increases. We also show how to solve horizontal noise problem in depth map. Horizontal noise in depth map appears when two lenses rectification not so precisely, or because of camera is too sensitive for given environment. In this work horizontal noise were affecting to detect two close located hands, which are located at the same altitude. To solve that problem we use masking method, and calculation of the mask given in this paper. Another solution can be the calibration [6], but it doesn't give always expected result, and already installed and fixed system cannot be calibrated all the time.

Differently with other existing algorithms we are not using previous frame in computations of current frame. Since keeping previous frame value and making additional comparison computations with current frame takes additional calculation times [7]. Fully independence from the previous frame was demonstrated in current algorithm.

This paper is organized as follows: In section II gesture recognition algorithm is mentioned. In Section III we explain how horizontal noise in a depth map appears and how it can be solved for our purpose task. Section IV is about two hand recognition and tracking, also it includes how grayscale histogram of the depth map can be used to detect two hands. Section $\mathrm{V}$ as a last section includes experiments and explanations about taken results.

\section{Gesture ReCOgnition Algorithm}

Stereo camera simulates the way human actually see and therefore creates the 3D effect when viewed. Depth map of the frame easily can be calculated using stereo camera. Depth map contains grayscale image where every pixel as a value contain $\mathrm{z}$ value of the pixel. Brighter pixels say that it close to camera than darker pixels.

In Our algorithm we will use 3D property of the image for the tracking hand and recognize gestures. Since our system can be used in systems for commanding using hand gesture. By analyzing where can be used command giving system we find out mainly commands will be given using outstretched hand or palm will be located in front of user. It means 
detected closest object usually will be human hand [8]. To be sure about detected region is human's hand it will be checked for skin color.

There might be some other objects in a camera view, but these static objects can be easily detected and masked out [9].

Stereo vision based hand tracking systems implies some threshold value for depth map. It will make an abstraction of invisible zone where entered object can be detected and tracked. The threshold of abstract zone can be fixed or it can be defined as a dynamic. In [5] shown how to define dynamic threshold based on grayscale histogram values of the depth map frame. Dynamic depth map threshold value makes system more flexible and motions performed by hand could be detected from different distances. In Fig. 1 the flowchart of depth threshold calculation algorithm is shown.

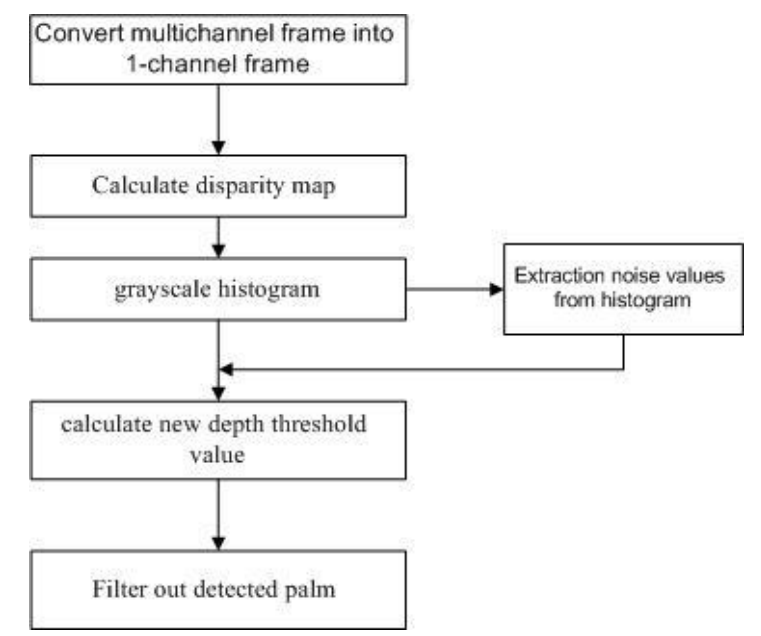

Fig. 1. Flowchart of the main algorithm.

After defining depth threshold the value of outstretched hand should be taken and then further operations can be run. Further algorithm of one hand gesture recognition system is described with details. Description starts from the step of receiving depth map, since this paper not devoted to depth map calculation.

Algorithm of one hand gesture recognition system:

1) Receiving depth map

2) Detecting and masking static objects in corners of frame

3) Defining filtering threshold using grayscale histogram of the image [6]

4) Labeling existing blobs in binary image

5) Filter out biggest blob or blob in certain range

6) Find skin-color in detected blob and make new blob based on skin detected information

7) Perform closing morphology operation over blob

8) Calculate centroid of the blob (x,y)

9) Refer to grayscale image and get $z$ coordinate of the blob or just get defined threshold value in step 3

10) Gesture detection, [Left, right, up, down, clockwise rotation, counter clockwise rotation, zig-zag]

11)

In step-5 developer can decide what is necessary for the system, because by step- 3 when hand is not outstretched full body of the subject will be detected and in step-5 it will be biggest blob. To avoid detection of full body and try to track it, developer can define some range for the blob. By statistical experience this range can be defined 300 3000, but it cannot be same all the time. Definition of the range is tricky and it should be configured based on deploying environment. Step- 6 is not considered as a mandatory and if usability of the system is fixed then this step can be excluded.

\section{HORIZONTAL NOISE IN A DEPTH MAP}

Dynamic programming helps to find a global optimum with a polynomial complexity, stereo methods that rely on it are not free from problems. The most severe limitation is imposed by the requirements of the uniqueness and ordering constraints which are not always fulfilled in real scenes. As a consequence errors can occur and it will appear as a horizontal streak in disparity map, accordingly in depth map. Appearing horizontal noise lines in depth map is common problem in stereo vision systems [10]. In our experimental camera that problem also appeared, and especially it was appearing when two outstretched hands were close to each other in a same altitude. To solve horizontal noise problem we use rectangular masking on that noise place, since noise is predictable in our case. In Fig.2. shown horizontal noise and how it affects on detecting two hands on same altitude.

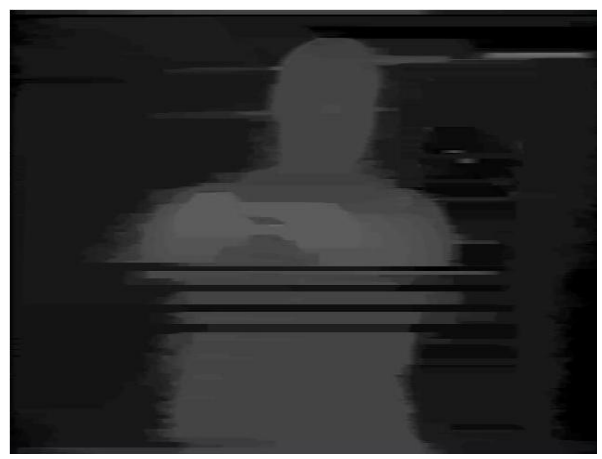

Fig. 2. Appearing horizontal noise on two close located hands on same altitude.

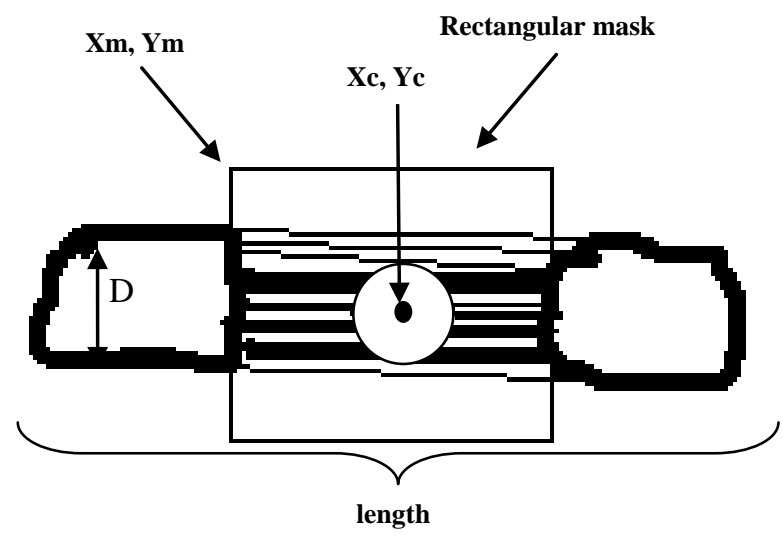

Fig. 3. Masking horizontal noise appeared in depth map

$$
\begin{gathered}
\text { mask.w = length }-2 D \\
\text { mask.h } h=D \\
X m=X c-\text { mask } \\
Y m=Y c-\text { mask }
\end{gathered}
$$


When Xm, Ym coordinates are calculated we can draw black rectangle area to mask out the noise place. The black rectangle area going to have width and height same order as mask.w and mask.h. As mentioned in Fig.3. horizontal noise appears in some special cases such as when two palms are located in same level and distance of the hands close to each other. When the hands are located in different altitude or their location far from each other even in same altitude then horizontal noise is not appearing.

\section{Two HAND RECOGNITION AND TRACKING}

In case of two hand detection, algorithm flow goes same as in one hand detection. When two hands are outstretched in the same way and same length, usual gesture recognition algorithm will have little difference in step-5. After labeling we should choose two appropriate blobs. In case of one hand detection algorithm we might skip skin-color detection step but for two hands recognition system it becomes mandatory, since second label might turn out pease of noise with bigger area. After detecting two palm blobs all other steps will be following one hand algorithm. As result value two $\mathrm{x}, \mathrm{y}, \mathrm{z}$ coordinates will be received. There are some experimental results in experiments and results section.

If two hands are not outstretched in a same way, above mentioned algorithm for two hands recognition cannot be applied without any changes. Second hand accidentally can be skipped, it can happen on step-3 when algorithm will check and detect first hand, but second hand which palms located little after first hand would be ignored. To avoid this case third step of the algorithm should be changed.

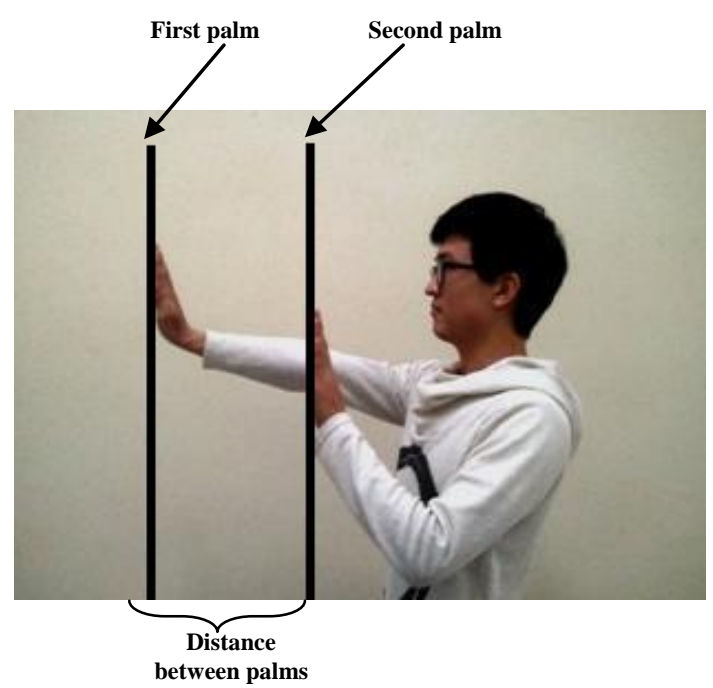

Fig. 4. Two different outstretched hands

Algorithm to find threshold value for two different outstretched hands, using grayscale histogram:

1) Detect first hand by [5]

2) Perform skin color detection over first detected hand

3) Continuously scan grayscale histogram, until next extreme point was found

4) Skin-color scanning in received blob

5) Checking blob region to not being face

This algorithm will define two threshold values for two different outstretched hands, using these threshold values possible to track both hands from any location.

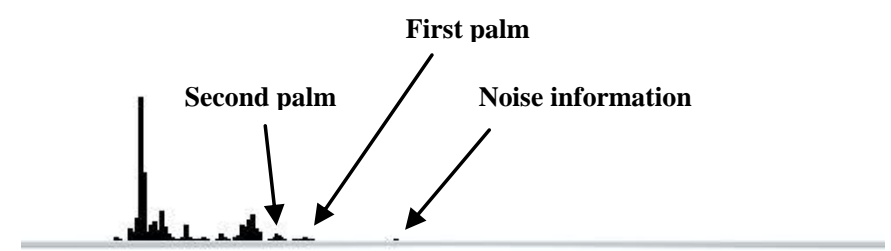

Fig. 5. Histogram value for the two different outstretched hands.

As shown in Fig.4. First palm is appearing in threshold where first extreme point in histogram appeared. Accordingly second extreme will point second palm detected threshold value. It means we should scan more compared with simple histogram grayscale threshold definition algorithm.

Two different length outstretched hands can give more opportunities and multi commands to the system. Especially if the $\mathrm{Z}$ value of the hands would be extracted and used for behavior detection it will add at least two time more different commands to command list.

\section{EXPERIMENTS AND RESULTS}

To implement and make some experiments over our algorithm we decide to use open source computer vision (OpenCV) library and VisionST(stereo camera with FPGA depth map calculation) stereo camera. This camera calculates depth map in hardware level, it gives advantage to any system where it will be used, since speed of the camera can reach 40 50 fps. Three output signals are coming from the Vision ST camera: left, right and depth map frames. In Fig. 8. given two hand detection algorithm results, in color image two hands were detected and occluded into rectangles.

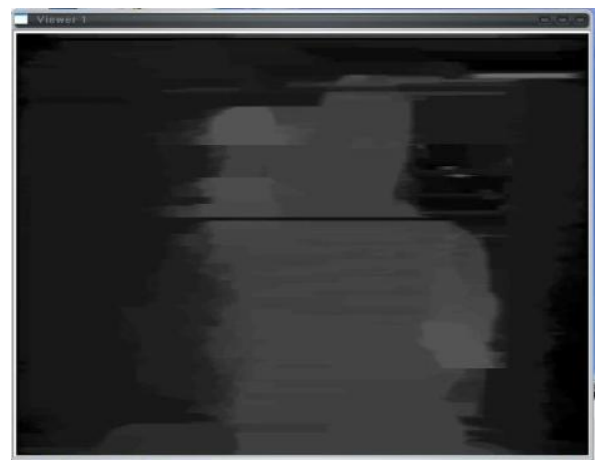

Fig. 6. Depth map of the two outstretched palms

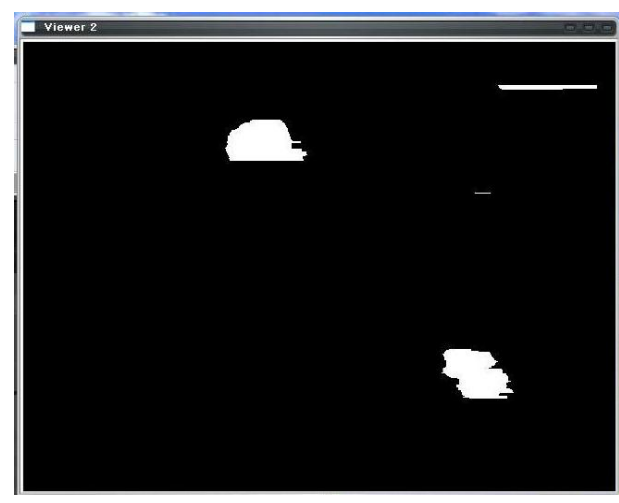

Fig. 7. filtered two outstretched palms 


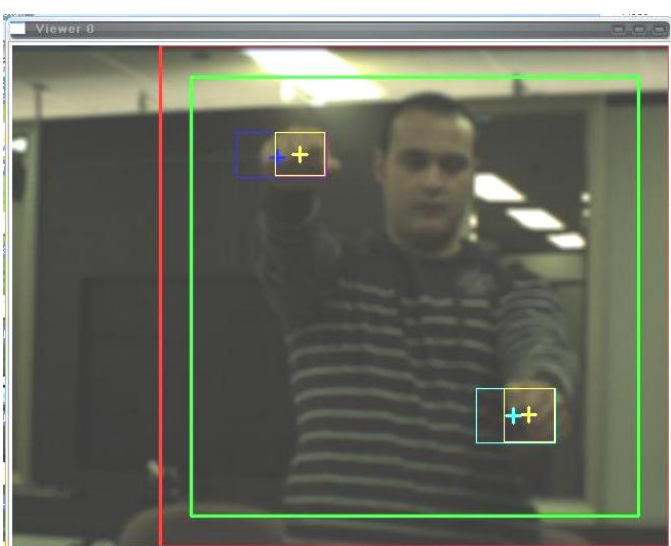

Fig. 8. Two hand detection process and detected hands occluded into rectangle.

From experiment images it's not difficult to detect that depth map calculation in hardware implementation works quite well, since it's not sensitive to high luminary lights. Horizontal noise can be easily detected from depth map of the image, especially where neighbour pixels having similar color range.

\section{CONCLUSION}

In this paper we suggested algorithm to tracking humans two hands based on grayscale image from stereo camera. Even though two hands are outstretched in different way we can detect them. This algorithm also includes how to solve problem with appeared horizontal noise. We suggest masking method to solve horizontal noise in depth map, which makes some mystifications in algorithm when two hands are getting close in same altitude. Masking method is calculates necessary rectangle area and positions of this mask rectangle. The main difference of our suggested method in this paper is , we are using grayscale histogram values of images to define threshold for image. From this approach subject whose hand should be tracked and recognized can be located in flexible distance from camera. This algorithm uses flexible threshold for the disparity map, it defines by grayscale histogram value. This algorithm can be easily implemented for the manipulating robots by hand movement. As a future work we planned to improve the accuracy of our method by adding some morphology detection methods.

\section{ACKNOWLEDGMENT}

This research was supported by Industrial Source Technology Development Programs and "The next generation core technology for Intelligent Information and electronics" project funded by the Ministry of Knowledge Economy (MKE) of Korea.

\section{REFERENCES}

[1] G. Lu, L. K. Shark, G. Hall, and U. Zeshan, "Hand motion recognition and visualisation for direct sign writing," 14th International Conference Information Visualisation, 2010.

[2] S. Jun, J. Park, C. Park, I. K. Jung, Y. O. Kim, and B. Kim, "Morphological Approach of Stereo Camera based Human Motion Capture System," Intelligent Mechatronics Research Center, Korea Electronics Technology Institute, Bucheon, Korea.

[3] H. An and D. Kim, Hand Gesture Recognition using 3D Depth Data.
[4] N. Jojic, M. Turk, and T. S. Huang, Tracking Self-Occluding Articulated Objects in Dense Disparity Maps, ECE Dept. and Beckman Institute University of Illinois at Urbana-Champaign.

[5] R. R. Igorevich, P. Park, D. Min, Y. J. Park, J. C. Choi, E. Choi, "Hand gesture recognition algorithm based on grayscale histogram of the image," 4th International Conference on Application of Information and Communication Technologies (AICT), 2010.

[6] K. Konolige and D. Beymer, SRI Small Vision System: User's Manual Software version 3.2g, Videre Design, November 2004.

[7] S. Jun, Stereo Camera based Human Motion Capture by using Expectation Maximization Algorithm, Dept. of Electrial and Electronics Engineering, The Graduate School of Yonsei Univ. 2006.

[8] W. You, H. Jiang, and Z. N. Li, "Real-time multiple object tracking in smart environments," in Proceedings of the 2008 IEEE International Conference on Robotics and Biomimetics Bangkok, Thailand, February $21-26,2009$

[9] D. Schreiber, "Generalizing the Lucas-Kanade algorithm for histogram-based tracking," Smart System Division, Austrian Research Centers GmbH - ARC, Donau-City-Strasse 1, A-1220 Vienna, Austria

[10] S. Palmisano, R. S. Allison, and I. P. Howard, Effects of horizontal and vertical additive disparity noise on stereoscopic corrugation detection, pp. 3133-3143, 2001.

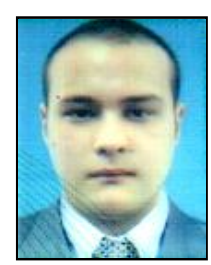

Rustam Rakhimov Igorevich Rakhimov Rustam Igorevich received the B.S of Information Security from the department of Information Technology of TUIT (Tashkent University of Information technology) in 2008, respectively. He's studying Computer Science and engineering in Konkuk University and at the same time having part time researcher job in Korea Electronics Technology Institute. He also completed the course of Young developers training and support center and some other courses like DBC (Diploma in Business Computing). $\mathrm{He}$ joined Linux localization project organized between TUIT and ETRI where he took part as leading programmer.

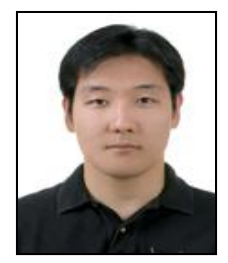

Pusik Park received B.S. and M.S. at School of Electronics, Telecommunication \& Computer Engineering, in Korea Aerospace University. He's been working for the Korea Electronics Technology Institute since 2002 and studying for a Ph.D.'s degree. Now he is working as a Senior Researcher in a SoC Research Center. Currently He is leading few projects funded by MKE (Ministry of Knowledge and Economy) Korea. Most of his projects are related to communications, such as AFDX and MOST communication networks.

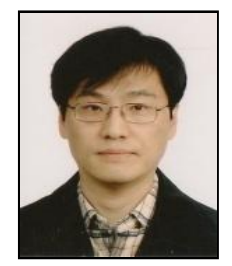

Choi Jongchan Jongchan Choi received B.S. at Department of Electronic Engineering in Kyugnhee Unversity, M.S. at Department of Computer Science in SeoKyeong University and Ph.D. at School of Information and Communication Engineering in Chungbuk National University. He' d been working for the Samsung SDI from 1985 to 1990 and has been heading the SoC Platform Research Center for the Korea Electronics Technology Institute till 2011. Since 2011 he had become a Vice President of System IC R\&D department in Korea Electronics Technology Institute. Currently he is supervising dozens of projects funded by MKE (Ministry of Knowledge and Economy) Korea.

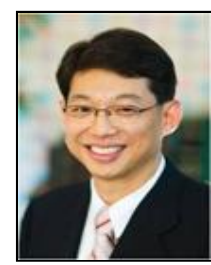

Dugki Min Dugki Min graduate M.S in Major of Computer Science at Michigan State University. Received his Ph.D. in Computer Science at Michigan State University 1991 1995. Vice President of International OMG Standard Organization KSIG. And also Professor, Distributed Multimedia System (DMS) Lab. Konkuk University. Vice president of Korea Society for Simulation, Software Architecture Modeling Research Group 2003 present. WSQM TC Chairman at Organization for the Advancement of Structured Information Standards (OASIS) 2005 Present. Pointed as Referencing Professor by Korea Software Technology Association (KOSTA) 2006 Present. 